\title{
Olanzapin hilft gegen Zytostatika-Erbrechen
}

\author{
Für das Neuroleptikum Olanzapin ergibt sich eine neue Indikation: Die Einnahme kann Übelkeit \\ und Erbrechen unter hochgradig emetogenen Chemotherapien wirkungsvoll verhindern.
}

\begin{abstract}
— Neben dem Haarausfall gehören Übelkeit und Erbrechen zu den Nebenwirkungen von Chemotherapien, welche die Lebensqualität erheblich beeinträchtigen und wesentlich zum Horror vor dieser Therapieform beitragen.

In einer randomisierten, doppelblind geführten Phase-III-Studie erhielten die Teilnehmer entweder das Neuroleptikum Olanzapin oder Placebo, jeweils in Kombination mit Dexamethason, Aprepitant oder Fosaprepitant und einem 5- $\mathrm{HT}_{3}$-Rezeptor-Antagonisten. Alle $\mathrm{Pa}$ tienten standen bereits unter einer Therapie mit Cisplatin in einer Dosierung $\geq 70 \mathrm{mg} / \mathrm{m}^{2}$ oder CyclophosphamidDoxorubicin.

Von den 380 aufgenommenen Patienten erhielten 192 an den Tagen 1-4 10 mg Olanzapin oral. 188 bekamen Placebo. Der Anteil von Patienten ohne Übel-
\end{abstract}

keit in den ersten 24 Stunden nach Applikation der Chemotherapie war in der Olanzapin-Gruppe mit 74\% signifikant größer als unter Placebo mit 24\%. Dieser Effekt setzte sich in statistisch signifikanter Weise über den Zeitraum von 25120 Stunden nach der Chemotherapie fort (42 vs. 25\%). Auch der sekundäre Endpunkt eines vollständigen Ansprechens - gar keine Übelkeit und keine Anwendung von Akutmedikamenten fiel mit 86 vs. $65 \%$ bzw. 67 vs. $52 \%$ für die genannten Zeiträume zugunsten von Olanzapin aus.

Die wichtigste Nebenwirkung von Olanzapin war ein sedierender Effekt, über den 5\% der Patienten in der Verumgruppe am Tag 2 berichteten.

- Navari RM, Qin R, Ruddy KJ et al. Olanzapine for the prevention of chemotherapy-induced nausea and vomiting. NEngl J Med. 2016;375:134-42

\section{KOMMENTAR}

Olanzapin ist ein Neuroleptikum, das multiple Neurotransmitter blockiert, darunter neben dem Dopaminrezeptoren auch mehrere Serotoninrezeptoren der Typen 5- $\mathrm{HT}_{2 \mathrm{~A}}$, 5-HT $2 \mathrm{C}, 5-\mathrm{HT}_{3}$ und 5-HT . Zusätzliche Wirkungen beziehen sich auf a1-Adrenozeptoren, Acetylcholin- und muskarinische Rezeptoren sowie $\mathrm{H}_{1}$-Rezeptoren im zentralen $\mathrm{Ner}$ vensystem. Man nimmt an, dass beim Mechanismus von Übelkeit und Erbrechen $D_{2}-$, 5- $\mathrm{HT}_{2 \mathrm{C}^{-}}$und 5-HT - -Rezeptoren die wichtigste Rolle spielen. Der eindrucksvolle Effekt von Olanzapin am ersten Tag nach der Chemotherapie nutzt sich zwar über die gesamte Beobachtungszeit hinweg betrachtet etwas $a b$-doch dürfte jeder einzelne Tag, den sie ohne oder nur mit leichter Übelkeit verbringen, von den betroffenen Patienten als wahrer Segen wahrgenommen werden.

Prof. Dr. med. H. S. FüeßI

\section{So sieht eine Nieswolke in Ultrazeitlupe aus}

Beim Niesen potenziell infektiöse Organismen herausgeschleudert. Welch gewaltige Geschwindigkeiten und Reichweiten dabei erzielt werden, zeigt diese Sequenz von Bildern in Abständen von $20 \mathrm{~ms}$. Sie stammen aus einem Hochgeschwindigkeitsvideo der Nieswolke einer

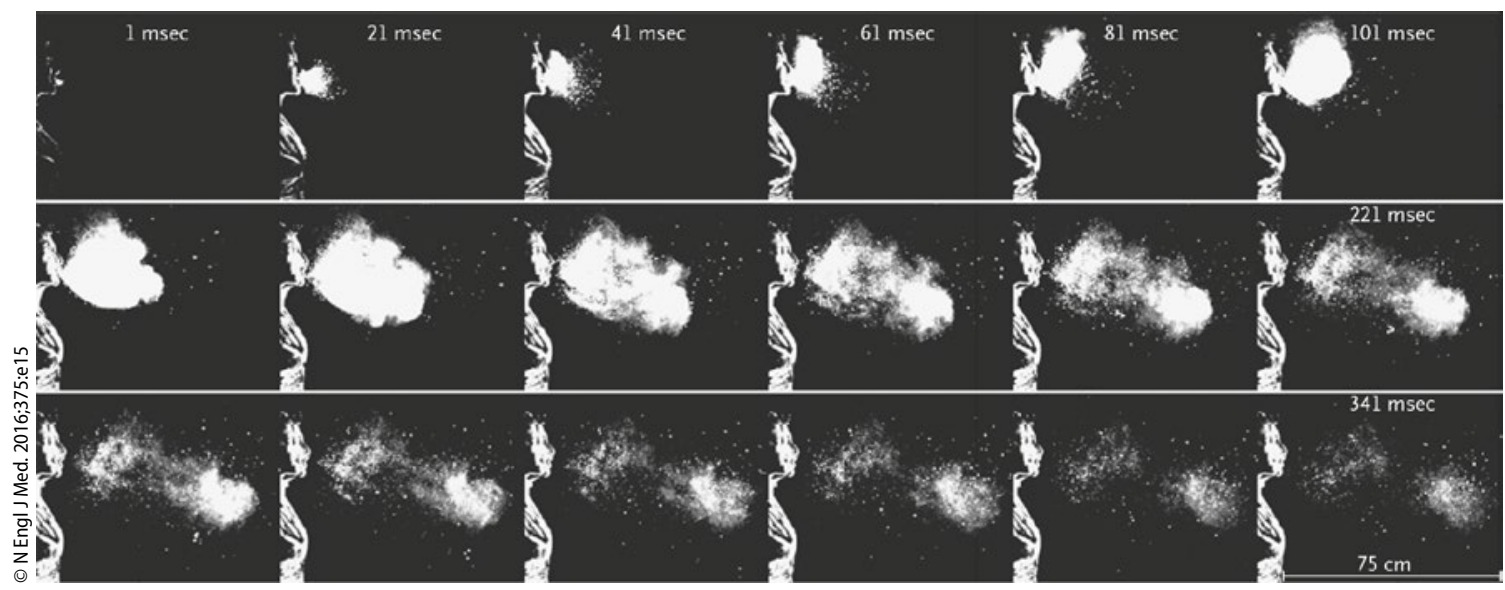

Ausbreitung einer Nieswolke binnen einer Drittelsekunde in 18 Bildern. Der Niesende steht jeweils links. gesunden Person. Man erkennt die Ausbreitung einer turbulenten Wolke, die aus heißer und feuchter Ausatemluft, Schleim- und Speichelfäden sowie Tröpfchen und Überresten von Tröpfchenkondensationskernen besteht. Die Ejektion dauert bis zu 150 ms (obere Reihe) und entwickelt sich dann zu einer inhomogenen, größeren Wolke mit turbulenten Strömungen. Die größten Tröpfchen können sich über eine Entfernung von 1-2 m ausbreiten. Kleinere Tröpfchen verbleiben in der Wolke und können noch Minuten später 6-8 $\mathrm{m}$ vom Nieser entfernt nachgewiesen werden.

Prof. Dr. med.H.S. FüeßI - Bourouiba L NEnglJ Med. 2016;375:e15 